\title{
DRAFT - HOW TO IMPROVE OPEN ROTOR AERODYNAMICS AT CRUISE AND TAKE-OFF
}

\author{
Cesare Hall, Alexios Zachariadis, Tobias Brandvik, Nishad Sohoni \\ cah1003@cam.ac.uk \\ University of Cambridge, Whittle Laboratory \\ $1 \mathrm{JJ}$ Thomson Avenue, Cambridge CB3 ODY
}

\begin{abstract}
A key challenge in open rotor design is getting the optimum aerodynamics at both the cruise and take-off conditions. This is particularly difficult because the operation and the requirements of an open rotor are very different at cruise compared to takeoff. This paper uses CFD results to explore the impact of various design changes on the cruise and take-off flow-fields. The paper then considers how a given open rotor design is best operated at take-off to minimise noise whilst maintaining high thrust. The main findings are that various design modifications can be applied to control the flow features that lead to lost efficiency at cruise and increased noise emission at take-off. A breakdown of the lost power terms from CFD solutions demonstrates how developments in open rotor design have led to reduced aerodynamic losses. At take-off, the operating point of the open rotor should be set such that the non-dimensional lift is as high as possible, without causing significant flow separation. This can be achieved through suitable amounts of re-pitch and speed up applied to a design. Comparisons with fully threedimensional CFD show that the amount of re-pitch required can be determined using simplified methods such as twodimensional CFD and a Blade Element Method.
\end{abstract}

\section{NOMENCLATURE}

\begin{tabular}{|c|c|}
\hline$a, a^{\prime}$ & Axial, Tangential induction factors \\
\hline$c$ & Blade Chord $[\mathrm{m}]$ \\
\hline$C_{L}, C_{D}$ & Lift Coefficient, Drag Coefficient \\
\hline$D$ & Diameter $[\mathrm{m}]$, Drag $[\mathrm{N}]$ \\
\hline$i$ & Incidence Angle \\
\hline$g$ & Axial spacing between rotors $[\mathrm{m}]$ \\
\hline$L$ & Lift $[\mathrm{N}]$ \\
\hline$\dot{m}$ & Mass flow rate $\left[\mathrm{kg} \mathrm{s}^{-1}\right]$ \\
\hline$M$ & Mach Number \\
\hline$N$ & Rotor blade count \\
\hline$p, p_{0}$ & Static, Stagnation pressure $[\mathrm{Pa}]$ \\
\hline$P_{\text {in }}$ & Input shaft power [W] \\
\hline$r, \Delta r$ & Radius [m], Radial thickness [m] \\
\hline$R$ & Rotor tip radius $[\mathrm{m}]$ \\
\hline$s$ & Specific entropy $\left[\mathrm{J} \mathrm{kg}^{-1} \mathrm{~K}^{-1}\right]$ \\
\hline$t$ & Section maximum thickness [m] \\
\hline$T$ & Temperature $[\mathrm{K}]$ \\
\hline$U$ & Blade speed $\left[\mathrm{m} \mathrm{s}^{-1}\right]$ \\
\hline$V$ & Absolute flow velocity $\left[\mathrm{m} \mathrm{s}^{-1}\right]$ \\
\hline$W$ & Relative flow velocity $\left[\mathrm{m} \mathrm{s}^{-1}\right]$ \\
\hline$w$ & Wake velocity defect $\left[\mathrm{m} \mathrm{s}^{-1}\right]$ \\
\hline$x$ & Axial (or Streamwise) distance [m] \\
\hline$X$ & Net thrust $[\mathrm{N}]$ \\
\hline
\end{tabular}

\begin{tabular}{ll}
$Y_{p}$ & Section loss coefficient \\
$\beta$ & Rotor pitch angle \\
$\delta$ & Wake width [m] \\
$\phi$ & Relative flow pitch angle \\
$\eta$ & Propeller Efficiency \\
$\rho$ & Density [kg m $\left.{ }^{-3}\right]$ \\
$\Omega$ & Rotational speed $\left[\mathrm{s}^{-1}\right]$ \\
Subscripts & \\
\hline 0 & Free stream value \\
1 & Inlet to front rotor \\
2 & Inlet to rear rotor \\
3 & Rear rotor exit \\
$a$ & Ambient conditions \\
$j$ & Far downstream (jet) \\
$F, R$ & Front Rotor, Rear Rotor \\
$r e l$ & Relative value \\
$x$ & Axial component \\
$\theta$ & Tangential component
\end{tabular}

\section{INTRODUCTION}

The aerodynamics of a contra-rotating open rotor operating at cruise are illustrated in Fig. 1, which is a schematic for a typical modern open rotor design. The key flow-features can be summarised as:

(i) High subsonic axial Mach numbers leading to transonic flow over the rotor blades with weak shocks and small regions of supersonic flow.

(ii) Minimal contraction of the propeller capture streamtube (the tip streamlines in the meridional view are at almost constant radius).

(iii) The rotors are set at a pitch angle close to the axial direction at a small incidence angle to the oncoming relative flow. This leads to thin rotor wakes and small tip vortices.

The key requirement at cruise is low specific fuel consumption and this is the main attraction of the open rotor concept. However low near-field cabin noise is also important and this is likely to be an area of increasing interest for future research. Low specific fuel consumption depends on achieving high propeller efficiency combined with a high thermal efficiency of the gas turbine. The thermal efficiency depends on the engine core technology and cycle thermodynamic design, and this will not be considered further here. The propeller efficiency is defined as:

$$
\eta=V_{0} X / P_{i n}
$$


This represents the ratio of total propulsive power to the aircraft (thrust $\times$ flight speed) to the shaft power input to the propellers (torquexrotational speed). The propeller efficiency can also be written in terms of the various sources of lost mechanical power as derived in the Appendix:

$$
\eta=1-\frac{\dot{m}}{P_{i n}}\left\{T_{a} \Delta s_{F}+T_{a} \Delta s_{R}+\frac{1}{2}\left(V_{j x}-V_{0}\right)^{2}+\frac{1}{2} V_{j \theta}^{2}\right\}
$$

Equation (2) shows us that the propeller efficiency is reduced by irreversible losses in each of the rotor blades, $T_{a} \Delta s_{F}+T_{a} \Delta s_{R}$, excess axial kinetic energy in the downstream jet, $\left(V_{j x}-V_{0}\right)^{2} / 2$, and residual swirling kinetic energy, $V_{j \theta}^{2} / 2$. The magnitudes of each of these sources of lost power are considered further in the next section.

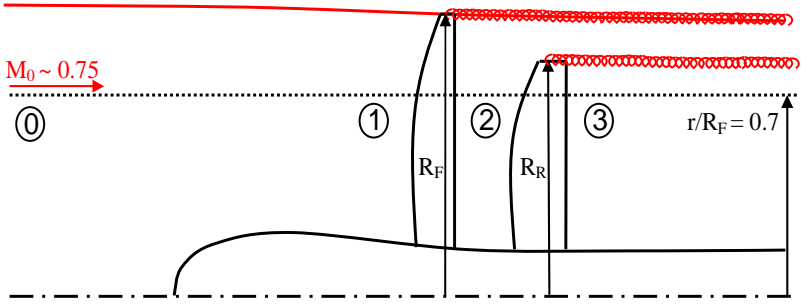

(a) Meridional view

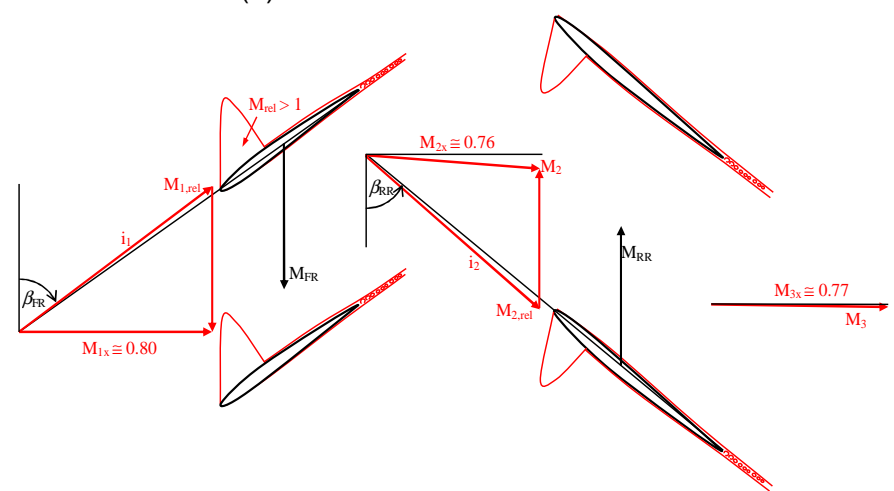

(b) Blade-to-blade view at $r / R_{F}=0.7$

Fig. 1 The open rotor cruise flow field

The aerodynamics at take-off are completely different to those at cruise. Figure 2 shows the key features of the take-off flow field, which can be summarised as follows:

(i) The flow is fully subsonic everywhere.

(ii) High contraction of the capture streamtube leading to increasing axial Mach number through the propeller rotors.

(iii) The rotors are set at a pitch angle close to the tangential direction at high incidence relative to the oncoming flow. This leads to thick rotor wakes and large tip vortex structures.

The key requirement at take-off is low community noise. However, the open rotor also needs to generate as much thrust as possible in order to get the aircraft airborne for the minimum size of engine. Therefore, the take-off design requirements can be summarised as minimum noise emission and maximum thrust per unit frontal area.

The noise from an open rotor is complex, because there are numerous different sources, each with different characteristics in terms of spectra and directivity, and each generated by different aerodynamic mechanisms. However, various previous studies such as [1], [2] and [3] led to some general principles that can be applied to diminish the key noise sources. Within this paper, these general principles will be applied to show how changes in the design and operation of an open rotor can modify the take-off aerodynamics in ways that are expected to reduce the noise emission.

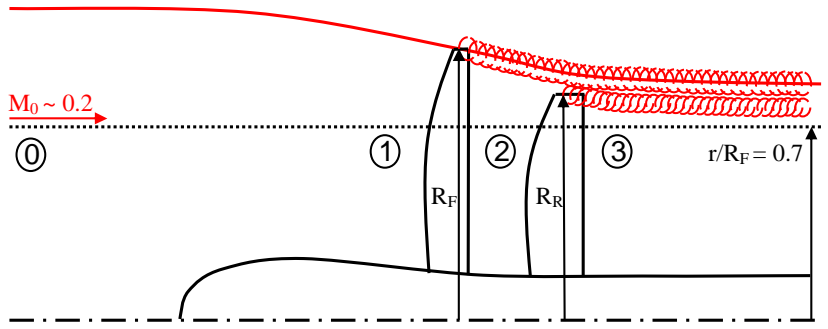

(a) Meridional view

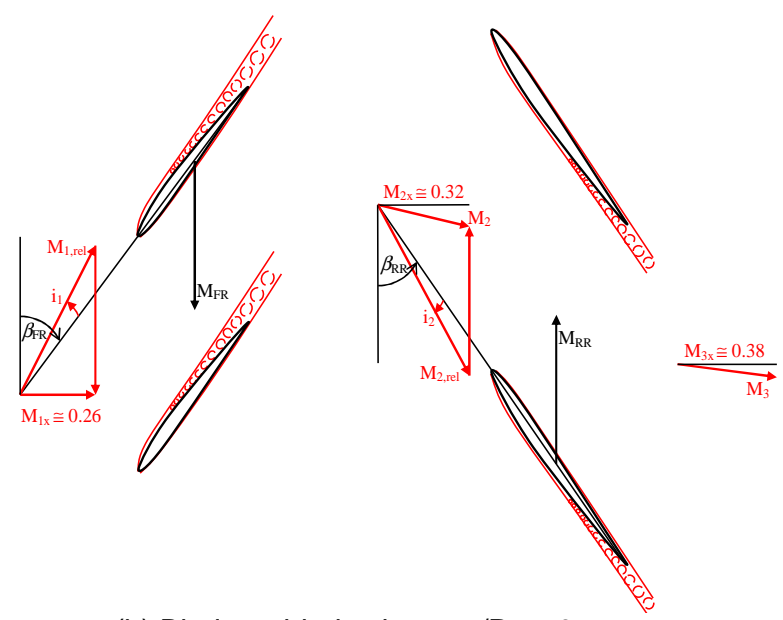

(b) Blade-to-blade view at $r / R_{F}=0.7$

Fig. 2 The open rotor take-off flow field

Historically, propellers were designed solely for maximum cruise efficiency [4]. Take-off was considered as an offdesign condition where the requirement was simply to generate sufficient thrust. However, in modern open rotor designs, because the noise emission is critical, both take-off and cruise are considered as design conditions.

Designs that satisfy the requirement for low noise at takeoff and high cruise efficiency have been explored in recent papers, see [5], [6] and [7], for example. In these studies, results from advanced CFD were combined with aeroacoustic methods in order to optimise the propeller geometries. Studies such as these have shown that various design modifications combined with changes in operating conditions have the potential to improve efficiency and reduce noise. However, because many modifications were made simultaneously the individual effects of each change are not clear. 
The approach taken in the first part of this paper is to draw on some recent computational studies of open rotor aerodynamics and noise, such as [8], [9] and [10] to explain the individual impact of several key open rotor design features on the aerodynamics at cruise and take-off. Since all noise originates in the flow-field, understanding the impact on the take-off aerodynamics can be used to infer the expected effects on the noise emission.

The second part of the paper examines take-off operational effects. The aim is to understand how, for a given design, the open rotor speeds and pitch angles should be set in order to minimise noise whilst maximising thrust per unit area. Some understanding of the effects of speed-up and re-pitch has already been covered in [9], but here it is shown that simpler methods such as a Blade Element Method and 2D CFD can be applied to determine the required operating point. Furthermore, the balance between the non-dimensional lift and blade speed is shown to be critical.

The main objectives of the current paper are to (i) improve the understanding of how the aerodynamics at take-off and cruise are modified by design changes, (ii) show how operational changes can be best applied at take-off and (iii) demonstrate that lower-order aerodynamic methods as well as stateof-the-art CFD are useful in open rotor design. It should be emphasised that the paper focuses on aerodynamics and is not intended to address in detail the mechanical or acoustic aspects.

The basis of this paper was presented at the Royal Aeronautical Society conference, Progress Towards Open Rotor Propulsion Technology, in November 2012. The paper should be of interest to engineers and researchers involved in open rotor aerodynamic design and performance.

\section{OPEN ROTOR DESIGN EFFECTS}

Table 1 compares some key geometric parameters of a basic open rotor design from the late 1980s with those of an advanced recent design. The configuration described as the early design within this paper is a test case known as 'Rig-140' fitted with straight blades, which was tested by Rolls-Royce plc and is specified in detail in [11]. The recent design example used here is one configuration from a model test rig called 'Rig145', developed by Rolls-Royce plc and detailed further in [10]. Although it is not the latest state-of-the-art, it is representative of the current generation of open rotor designs

\begin{tabular}{|l|c|c|}
\hline Geometric Parameters & Early Design (1988) & Recent Design (2008) \\
Front Rotor HTR & 0.32 & 0.34 \\
Rotor Axial Spacing $\left(g / D_{\mathrm{F}}\right)$ & 0.21 & 0.31 \\
Rear Rotor Crop & No & Yes \\
Front rotor blades, $N_{F}$ & 7 & 12 \\
Rear rotor blades, $N_{R}$ & 7 & 9 \\
Side Views (not to scale) & & \\
& & \\
\hline
\end{tabular}

Table 1 Comparison of early and recent open rotor designs

Table 1 highlights some important developments made in open rotor design over the last 25 years. Firstly, recent designs use a combination of rear rotor crop and rotor-rotor axial spac- ing to manage interaction noise. Secondly, the numbers of front and rear rotor blades are different with more front rotor blades due to the higher loadings on the front rotor. Thirdly, the designs of front and rear rotors are completely distinct with different chord distributions, section designs and sweep variations.

Computational studies were performed for the designs illustrated in Table 1 and the CFD solutions were used to calculate all the terms in eqn. (2). The results are given in Table 2. In all cases, the CFD methodology was based on [11] using the Spallart-Allmaras turbulence model presented in [12]. Note that the pitch settings and rotational speeds used for the recent design are not intended to be precise matches of those that would be used in practice for take-off and cruise operation.

Table 2 shows that the early design has good overall efficiency at cruise. The losses in the front and rear rotors are significant, but the lost power due to excess kinetic energy and residual swirl in the jet are small compared to take-off. Note that the calculation of the residual swirl power is covered below. At take-off, the loss in the front rotor is high. This is because at this take-off operating condition the front rotor is highly separated and inefficient (see later). The excess kinetic energy loss is also high because at take-off the forward speed is low and the thrust is high, giving a large difference between the jet velocity and the flight speed. The swirl losses are also high due to poor matching of the front and rear rotors.

For the recent design the efficiency is higher at cruise and take-off. At cruise the power breakdown is similar to the early design, but with lower losses in the rotors and, despite a cropped rear blade (see below), only slightly higher swirl losses. However, at take-off, the rotor losses are much lower than the early design and are also lower than the losses in the current design at cruise. This is not surprising as modern designs are, of necessity, optimised for both the cruise and takeoff conditions. The excess kinetic energy losses are higher in the current design because, as shown by eqn. (A.2) in the Appendix, these losses increase with propeller efficiency for a given operating condition.

Note that in all cases, the values given in Table 2 do not quite add up to $100 \%$. This is because nacelle drag, spurious numerical losses, and non-uniform static pressure at the domain exit, are not included in the extraction of the power terms from the CFD solutions.

\begin{tabular}{|c|c|c|c|c|c|}
\hline & $X V_{0}$ & $\dot{m} T_{a} \Delta s_{F}$ & $\dot{m} T_{a} \Delta s_{R}$ & $\frac{1}{2} \dot{m}\left(V_{j x}-V_{0}\right)^{2}$ & $\frac{1}{2} V_{j \theta}^{2}$ \\
\hline Early Design & & & & & \\
CRUISE & $83 \%$ & $5.5 \%$ & $6.0 \%$ & $2.5 \%$ & $0.8 \%$ \\
TAKE-OFF & $55 \%$ & $9.5 \%$ & $4.0 \%$ & $22.0 \%$ & $3.5 \%$ \\
\hline Recent Design & & & & & \\
CRUISE & $86 \%$ & $4.8 \%$ & $4.4 \%$ & $2.7 \%$ & $0.9 \%$ \\
TAKE-OFF & $64 \%$ & $3.5 \%$ & $3.0 \%$ & $24.9 \%$ & $1.6 \%$ \\
\hline
\end{tabular}

Table 2 Power breakdown for early and recent open rotors

The remainder of this section considers some open rotor propeller design features and aims to explain how each one affects the aerodynamics at both cruise and take-off. The design features considered are: (i) rear rotor crop (ii) rotor-rotor axial 
spacing (iii) rotor sweep and (iv) rotor section geometry. These four design aspects have been chosen as they are regularly applied in modern open rotor designs and they are known to have significant implications to both cruise performance and take-off noise. In each case the designs shown in Table 1 are used as the test cases to demonstrate the design effects. Note that the rotor blade count is considered with rotor section geometry.

\section{Rear rotor crop}

One of the key principles of a contra-rotating open rotor is that the rear rotor removes the swirl put into the flow by the front rotor. This minimises the lost power due to the swirling kinetic energy in the jet behind the propeller, represented by the last term in Eqn. (2). Applying rear rotor crop means that flow that passes through the tip region of the front rotor passes over the tip of the rear rotor. Hence, some swirl imparted to the flow by the front rotor tip remains as residual swirl downstream of the engine and this leads to a loss of efficiency.

Figure 3 shows 3D CFD results of computed swirl angle distributions for designs with and without crop operating at cruise. The distributions are plotted for axial locations downstream of the front rotor (station 2) and far downstream of both rotors (jet). With crop there is a significant region of residual swirling flow near the front rotor tip radius. At lower radii, the residual swirl in the jet is very close to zero and the rear rotor design has effectively removed the front rotor swirl. In the design without crop, there is a small amount of residual swirl throughout the propeller span. This is due to the propellers in this configuration being identical (Table 1), combined with higher input shaft power to the front rotor.

Given that the exit flow is non-uniform, the loss in propeller efficiency due to residual swirl can be written as:

$$
\Delta \eta=-\frac{1}{2 P_{i n}} \int V_{j \theta}^{2} d \dot{m}=-\frac{\pi}{P_{i n}} \int_{0}^{R_{F}} \rho V_{j x}^{3} \tan ^{2}\left(\alpha_{j}\right) r d r
$$

This shows that the efficiency loss scales with the tangent of the residual swirl angle squared, and it is therefore essential to minimise this. Equation (3) was evaluated using numerical integration for the swirl distribution cases shown in Fig. 3 and in the cropped case, the residual swirl contributes a $0.9 \%$ loss in efficiency. For the early design without crop, the residual swirl accounts for a loss of $0.8 \%$, but this would be reduced in a modern design.

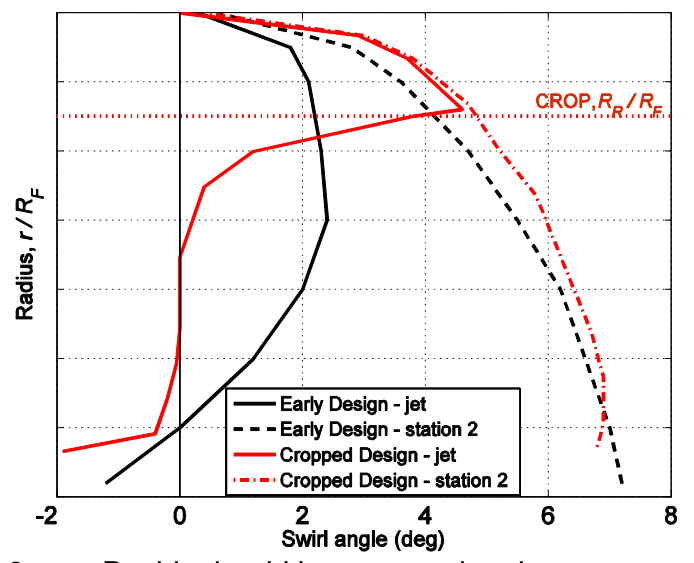

Fig. 3 Residual swirl in a cropped and non-cropped design
The motivation for applying crop is to reduce the noise at take-off caused by the interaction of the front tip vortex with the rear rotor, see [2] or [13]. Figure 4 shows a CFD result for a recent open rotor design operating at take-off with a cropped rear rotor. The computation is steady, which means that the front rotor tip vortex is smeared circumferentially when it passes through the mixing plane between the two rotors. However, it is clear that this smeared-out vortex passes above the rear rotor tip and the rear rotor tip vortex is radially inboard. In reality, due to the finite size of the tip vortices, there could still be interaction between the vortices and potentially some associated noise, see also [14]. There is therefore a question of how much crop is sufficient to effectively eliminate the vortex interaction noise. This can only be reliably determined through testing or with full unsteady CFD computations, such as those presented in [10].

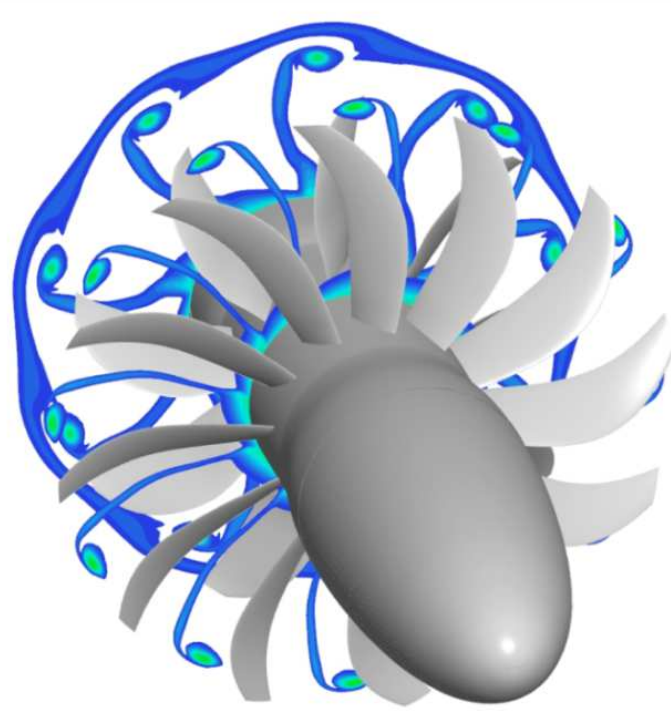

Fig. 4 Predicted contours of entropy for the cropped recent open rotor design operating at take-off

\section{Rotor-rotor axial spacing}

The further apart the front and rear rotors are placed, the more rear rotor crop is needed to avoid tip vortex interaction at take-off. This is shown by Fig. 5, taken from [10], which shows the trajectories of the front tip vortex at take-off, extracted from unsteady CFD solutions.

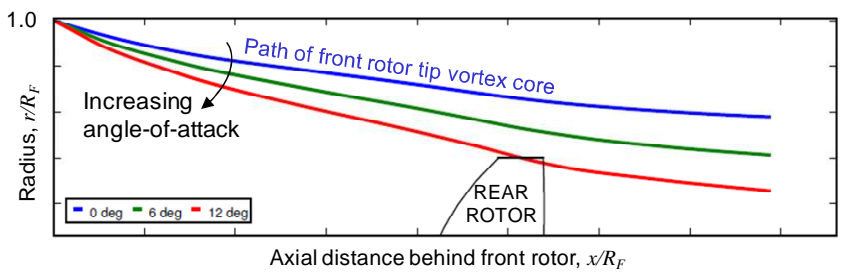

Fig. 5 Vortex trajectory from the front rotor of the recent design at different angles-of-attack, from [10]

Figure 5 demonstrates how the tip vortex moves inwards with downstream distance. In addition, when angle-of-attack 
effects are included, front rotor tip vortices that originate at the bottom of the engine move further inboard as they convect downstream. Thus, if the rotors are placed further apart, the crop required increases dramatically, further reducing cruise performance. In addition, any additional axial length will increase the overall engine weight and nacelle drag.

Increased axial gap between the rotors provides more distance for the front rotor wake to dissipate before it hits the rear rotor and it is mainly the characteristics of this wake impacting the rear rotor that determine the viscous interaction noise, see [13]. Larger spacings are also favourable for lowering potential interaction.

Figure 6 shows the variation in wake velocity deficit, $w / W$, with distance downstream of the front rotor for a wake model based on Schlichting [15] and for steady CFD results run on a front rotor alone configuration of the early design. A discussion of various classical wake models and their application to contra-rotating propellers, is given in chapter 5 of [16]. It's worth noting that a range of models are also derived in [16] for application to the forward and rearward potential field interactions between the two blade rows. Under certain conditions these potential field interactions can dominate [17].

The simple Schlichting model is derived from Prandtl's mixing length theory and can be summarised as:

$$
\frac{w}{W}=k_{1} \sqrt{C_{D} \frac{c}{x}} \text { and } \frac{\delta}{c}=k_{2} \sqrt{C_{D} \frac{x}{c}}
$$

, where $k_{1}$ and $k_{2}$ are constants.

In all cases the wake deficit decays and the wake width increases with increasing axial distance, which are both expected to reduce noise. Various wake decay models were also considered in [8] but the simple Schlichting model in Eqn. (4) was found to be the most promising in comparisons with CFD.

A key problem is that real open rotor wake characteristics are not well known. More rig experiments are needed that measure open rotor wake development and dissipation. Such tests could be used to validate computational results and to develop improved models of wake-blade interaction. Several studies present computed viscous wake interaction noise, but none of these have demonstrated that the wakes impacting on the rear rotors are correctly reproduced.

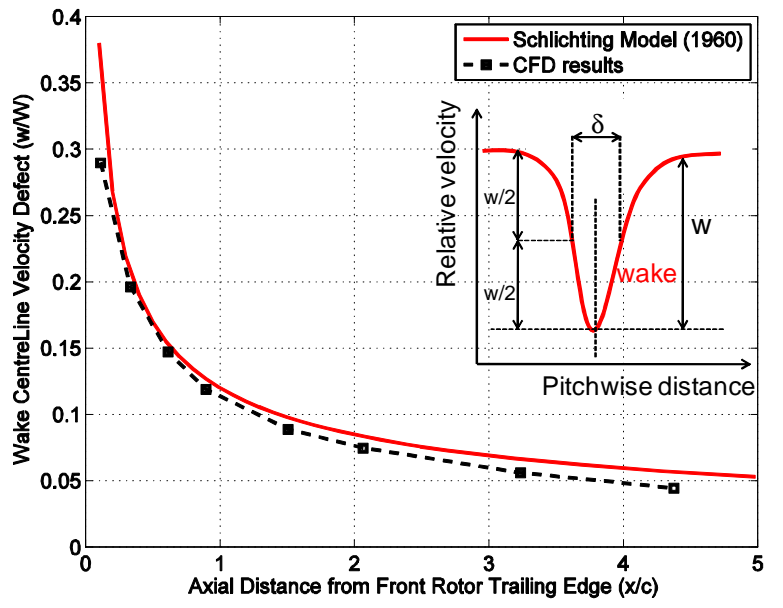

Fig. 6 Variation in take-off front rotor wake velocity deficit for Schlichting model and CFD.

\section{Rotor sweep}

Blade sweep relieves any transonic effects at high relative Mach numbers. At cruise, Fig. 7 demonstrates that without sweep, there is a supersonic region on the suction surface of both rotors, ending in a weak shock. With sweep added, the Mach number levels are reduced and the shock is eliminated. Any shock wave on the rotor will reduce efficiency through the shock itself and through increased loss in the rotor boundary layer. In addition, any shock waves introduce additional noise sources, which could increase cruise cabin noise.

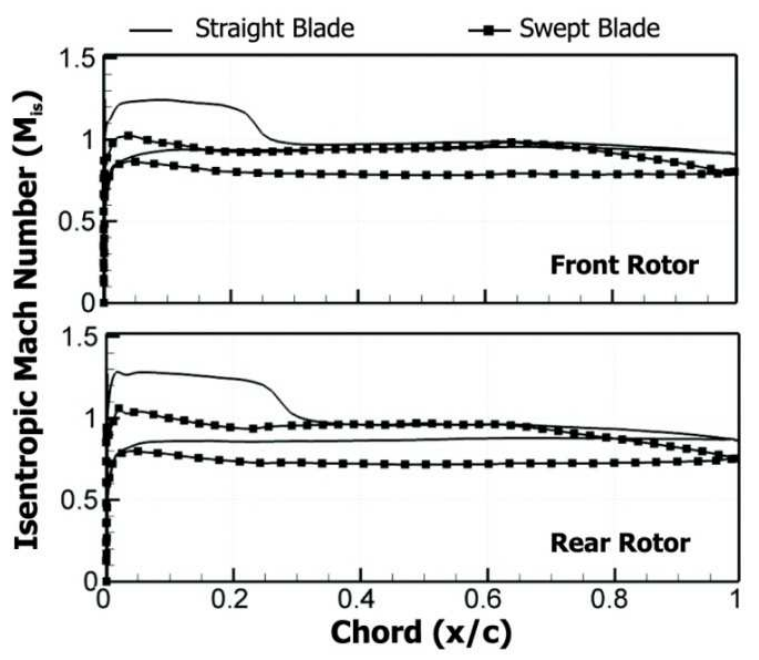

Fig. 7 CFD predicted section aerodynamics at $r / R=0.7$ for an early swept and unswept rotor blade at cruise.

The effect of applying sweep has potential benefits at takeoff. Figure 8 shows predicted surface streamlines for early designs of swept and unswept rotors for identical take-off conditions. In both cases a leading edge separation forms above about $30 \%$ span. In the swept blade case, the separated region is smaller and moves more radially outwards giving a smaller loss core at exit and corresponding higher efficiencies than the straight blade. In the case shown the sweep does, however, also give a larger hub separation, which will reduce its benefit.

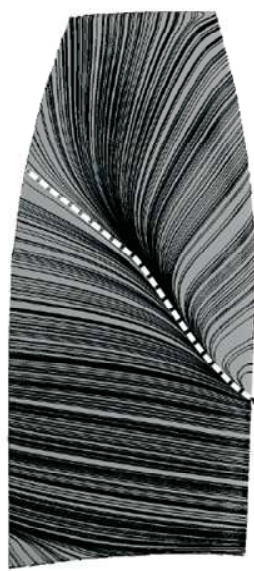

a) Straight Blade

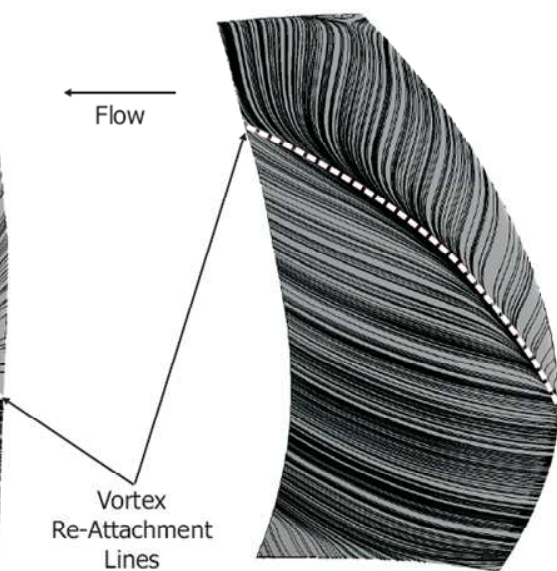

b) Swept Blade
Fig. 8 Comparison of suction surface streamlines at take-off. 
The development of the loss core downstream of the front rotor for the swept and unswept cases are shown in Fig. 9. For the straight blade the blade leading edge vortex and tip vortex are fairly distinct and cause a large region of loss. In the swept case, the leading edge vortex and tip vortex combine and interact within a smaller high loss region. It is expected that this loss core from the swept blade will lead to reduced interaction noise, although this has not been calculated.

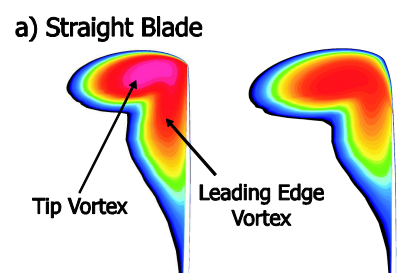

b) Swept Blade
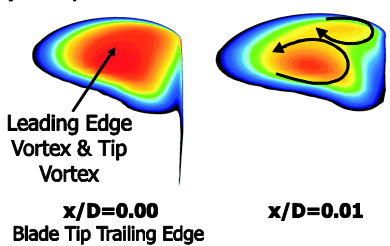

$x / D=0.01$
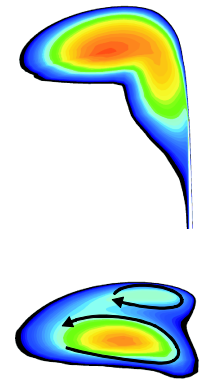

$x / D=0.02$
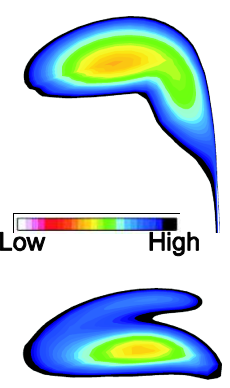

$\mathrm{x} / \mathrm{D}=\mathbf{0 . 0 3}$
Fig. 9 Comparison of take-off tip flow development downstream of a straight and swept rotor blade.

Other studies, such as [18] and [19], have found possible acoustic benefits from sweep. These identified how sweep introduces phase differences into the noise signals radiating from each spanwise part of a propeller. The balance and directivity of propeller noise sources can be modified by sweep, as demonstrated in [20]. Analytical work in [21] also showed that the precise radial distribution of sweep is important as it can lead not just to reductions but also, under certain conditions, to enhancement of the radiated tones.

\section{Rotor section geometry}

The 2D sectional performance of open rotors seems to have received surprisingly little attention. Many earlier designs were based on standard NACA aerofoils, see for example, [2] and [22], and there has been little published detail of the thickness and camber distributions for recent open rotor section designs. Here, we consider the cruise and take-off aerodynamic effects of some basic section geometric parameters.

\section{(i) Blade count and pitch-to-chord ratio}

A higher blade count, for a fixed rotor chord, reduces the loading on each propeller blade and this should give an efficiency benefit at cruise. However, increased blade count implies greater total wetted area and high blockage at the hub, which will tend to reduce efficiency. An optimum pitch-tochord ratio is expected to exist that gives low loss and sufficient operating range.

The blade counts for each rotor are critical to the noise since these determine the blade passing and interaction frequencies. It is generally accepted that it is favourable to have fairly high numbers on the front and rear rotors that will not generate common frequencies [23]. Adding more blades in- creases the number of noise sources, but this can be offset by reducing the blade chord.

Reduced chord (or increased aspect ratio) is expected to lower interaction noise and the rotor spacing required. However, the choice of chord must be appropriate to prevent any aeromechanical issues, such as flutter, and to achieve sufficient thrust at take-off. The number of blades that can be fitted are also limited by mechanical issues such as the design of the disk and the pitch change mechanism.

\section{(ii) Maximum thickness}

The maximum thickness of propeller aerofoil sections is usually dictated by mechanical requirements such as robustness to impact and aeromechanical response. Thicker sections will inevitably lead to higher transonic losses at cruise and lower efficiency. In addition, the thickness noise from a propeller blade scales with the volume displaced and therefore thicker sections tend to have greater rotor alone noise. Increased thickness can give an advantage at take-off though, because thicker sections tend to be more incidence tolerant (see below).

\section{(iii) Camber}

Increasing blade camber (or turning) can be used to increase the power input to the flow at take-off, without requiring high incidence onto the blade sections. This can lead to lower loss, because the flow is less likely to separate at the leading edge, giving a much cleaner take-off flow-field. Lower rotor loss from the front rotor implies smaller wakes and tip vortices, thus helping to reduce interaction noise.

At cruise, however, the flow turning required is much lower, because the loading is much less than at take-off. Highly cambered blade sections can therefore end up operating at negative incidence with higher cruise losses than low camber sections operating at their optimum cruise incidence.

\section{(iii) Leading edge profile}

Leading edge shape has been shown to influence minimum loss and operating range in compressor blades [24] and this is an area with potential for open rotors. The aim should be to prescribe robust leading edge shapes that will give low loss and the greatest possible incidence tolerance.

Figure 10 shows some results from 2D CFD predictions using MISES [25] for three different designs of open rotor section at take-off. MISES is a coupled inviscid method and boundary layer solver for turbomachinery cascades. It can therefore resolve the $2 \mathrm{D}$ viscous and transonic loss sources. The section in black, called the datum design, is a transonic section from an early open rotor. The dashed red section is an increased camber version of this. The solid red section is a thickened version of the datum design with a higher blade count (reduced pitch).

The plot in Fig. 10(b) shows loss versus incidence for these sections. The circles represent operating points where the required take-off thrust is achieved. The datum design is based on a section from the early design in Table 1 . This is at high incidence at take-off, and thus is predicted to have high loss. It is actually found to be separated in 3D CFD (see later). With increased camber, because incidence is defined here relative to 
the section stagger line (rather than the leading edge metal angle), the low-loss region moves to the right of the plot. The operating point needed for take-off thrust is now at much lower loss than the datum condition. Unfortunately, at cruise, this section is expected to operate at negative, non-optimum, incidence. The section with increased thickness and greater blade count has a much wider low loss region. The added incidence tolerance means the take-off operating point is not at high-loss, but the minimum loss is higher than the other sections. The cruise loss, although not shown here, is also predicted to increase due to the extra thickness and greater wetted area.

The results in Fig. 10 demonstrate that the propeller section designs can have a significant effect on the take-off aerodynamics and that a 2D method could be used to develop section design with low loss at both cruise and take-off.

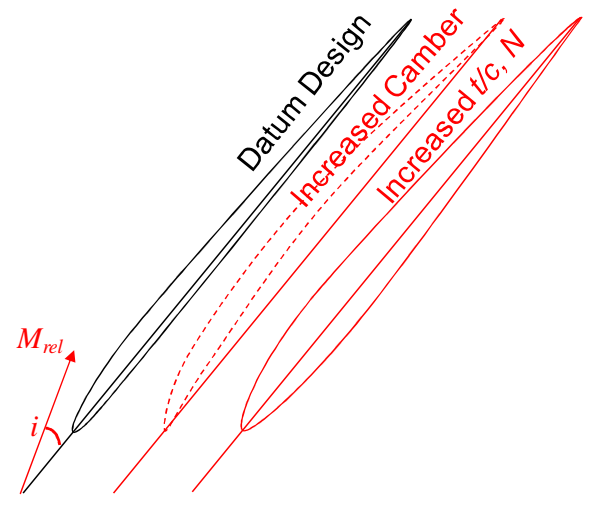

(a) Geometry of the three section designs

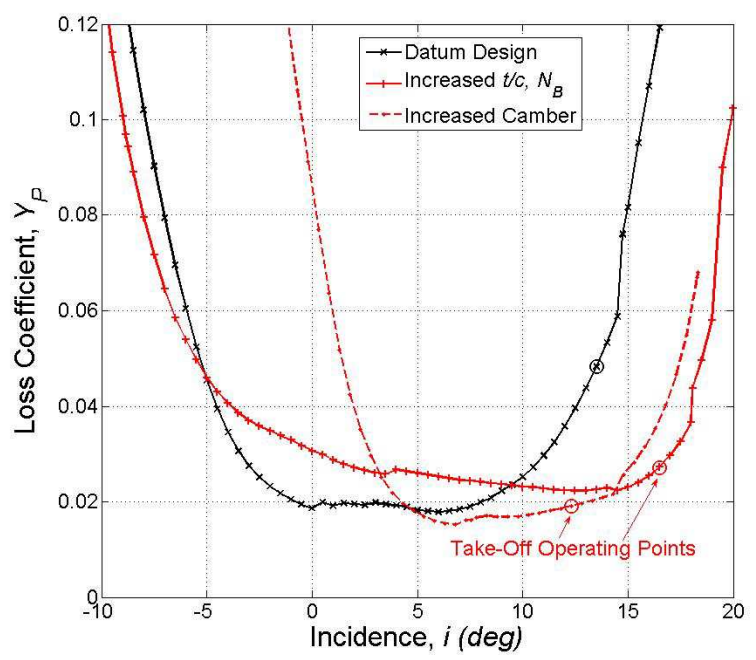

(b) Loss loops for the three section designs.

Fig. 10 Study of section performance at take-off using the 2D CFD code, MISES.

\section{TAKE-OFF OPERATION}

There is some useful flexibility in how contra-rotating open rotors can be operated at take-off. Although the torque ratio between the front and rear rotors is fixed by the gearbox, the variable pitch mechanisms for the rotors are independent. This means there are a range of pitch angles and rotational speeds that are possible for a given thrust requirement.
Propeller aerofoil aerodynamics can be considered in a similar way to aircraft wing sections. Figure 11 shows a typical lift coefficient-incidence plot for a propeller. Similarly to a wing, at take-off, high $C_{L}$ and incidence are required to give high lift (and therefore thrust) for a fixed lifting area. However, the lift coefficient must not be so high such that separation and stall occurs as this will lead to high noise and poor performance, as well as being potentially dangerous. At cruise operation, low incidence is required to give the maximum Lift-toDrag ratio, $L / D$.

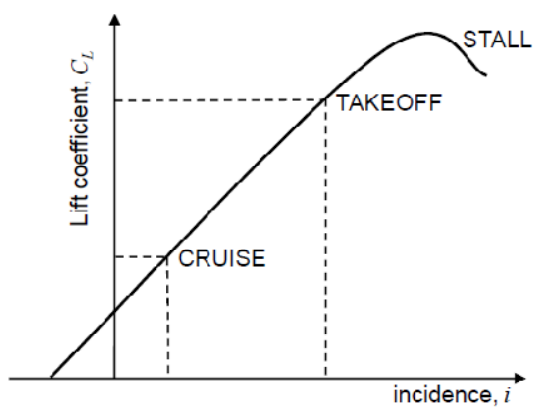

Fig. 11 Lift-incidence curve for an aerofoil

In this section we consider how computational methods can be used to determine a take-off condition that gives the required thrust with improved aerodynamics. Only the front rotor aerodynamics are considered in detail and it is assumed that the open rotor geometry and gearbox are fixed.

\section{Blade Element Method}

A simple Blade Element Method (BEM) can be used to explore and understand the effects of varying blade pitch and speed. The BEM used here is based on Glauert (1926) for a single rotation propeller [26]. Figure 12 shows the nomenclature and angle convention used for a blade element.

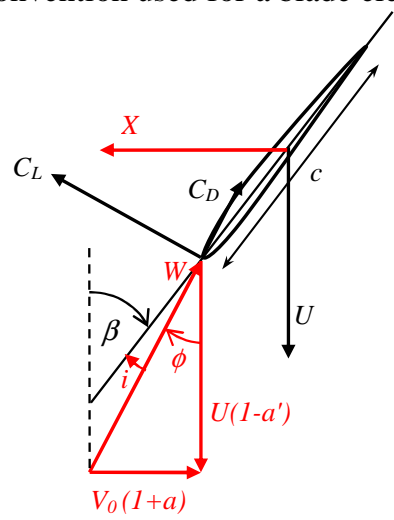

Fig. 12 Velocity triangle and aerodynamic forces for a propeller blade element

In this method, the lift coefficient is simply assumed to be linearly proportional to incidence, i.e. $C_{L}=k_{L} i$. The section drag coefficient is assumed to be related to the lift coefficient by $C_{D}=C_{D 0}+k_{D} C_{L}^{2}$. The constants, $k_{L}, C_{D O}$ and $k_{D}$ were chosen to give a typical, realistic lift-drag relationship for an open 
rotor propeller. The axial and tangential induction factors, $a$ and $a^{\prime}$ are critical within a BEM. These depend on the lift and drag coefficients on the blade element and therefore the method iterates to find the inlet velocity triangle that is consistent with the computed values of $C_{L}$ and $C_{D}$. The thrust on the blade element is given by resolving the forces in Fig. 12 in the axial direction:

$$
X=\left(\cos \phi-\frac{C_{D}}{C_{L}} \sin \phi\right) \frac{1}{2} \rho c \Delta r W^{2} C_{L}
$$

At take-off, the required total thrust from an open rotor is fixed by the aircraft requirement. Equation 5 shows that thrust increases as the pitch angle, $\phi$, reduces, and as the relative flow speed, $W$, the blade element area, $c \Delta r$, and the lift coefficient, $C_{L}$ increase. Therefore, for a given thrust and propeller size, it is possible to operate an open rotor blade at a lower lift coefficient, by repitching the blade to reduce the incidence whilst simultaneously increasing the blade speed $U$ (and thereby raising $W$ ).

In Fig. 13 results from the BEM and 3D CFD are compared for a range of take-off conditions, all at the same propeller thrust. These results are for the front rotor of the early design of open rotor shown in Table 1. It is worth noting that the BEM takes less than 1s to run the full range of operating conditions shown. The CFD results are from [9] and each computation took about 1 day of computational time.

As the blade is re-pitched, the incidence drops, reducing the lift coefficient. To recover the thrust, the rotational speed must increase, which, as shown by the velocity triangle in Fig. 12 , acts to increase the incidence. The resultant reduction in incidence is therefore less than the change in rotor pitch angle (the top plot in Fig. 13 shows that re-pitching by $-20^{\circ}$ gives an incidence reduction of only $7.5^{\circ}$ ). The fractional increase in rotational speed required is considerable, but much less than the corresponding decrease in incidence. As shown by the middle plot in Fig. 13, as the incidence reduces by a factor of 2.5, the rotational speed increases by a factor of about 1.58 . Note that $1.58^{2} \cong 2.5$ and from eqn. (6) it is expected that $X \propto U^{2} i$.
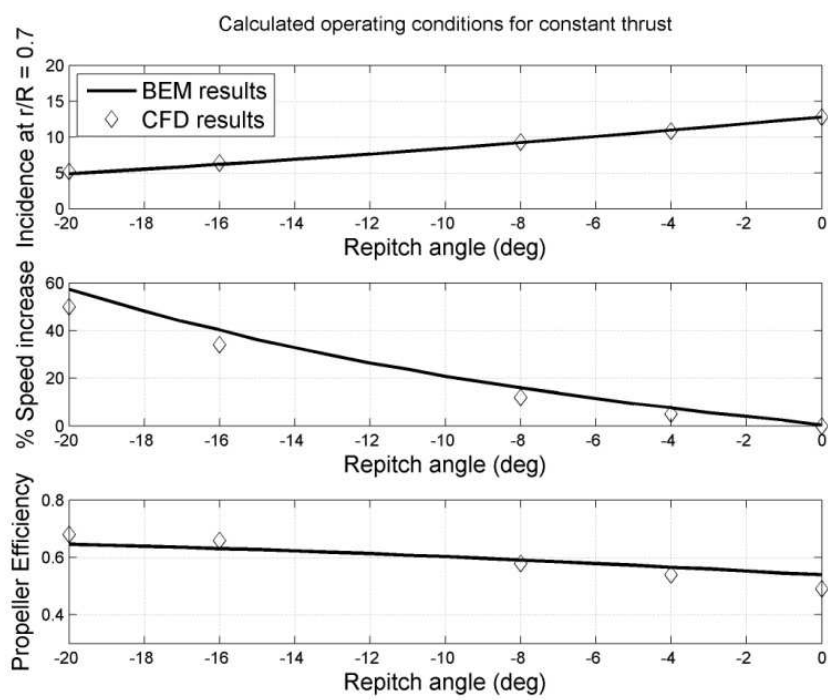

Fig. 13 Calculated operating conditions for constant thrust

As the incidence is reduced, both the CFD and the simple BEM predict improved efficiency. This is because $C_{L}$, and therefore $C_{D}$, are both reducing. The re-pitching and speeding up of the propeller is effectively moving the operating point down the $C_{L}-i$ curve in Fig 11. For the early design of propeller considered, the operating condition with no repitch is operating at high $C_{L}$ with separated flow and low efficiency (see below). It is therefore unsurprising that the efficiency continuously improves as repitch is applied. The difference in the efficiency variation between the BEM and CFD occurs because the BEM does not account for any separation or other 3D losses. The otherwise good agreement between the BEM and CFD results is to be expected since the BEM correctly determines the velocity triangle at inlet to the propeller and this is the main factor influencing the performance.

\section{Three-dimensional effects}

Fig. 14 shows some of the detailed 3D flow-field predicted by steady $3 \mathrm{D}$ CFD for the front rotor of the early open rotor design at the same take-off operating points considered above. As the rotor is re-pitched, reducing the incidence, the separation at the leading edge cleans up and the flow becomes more 2D.

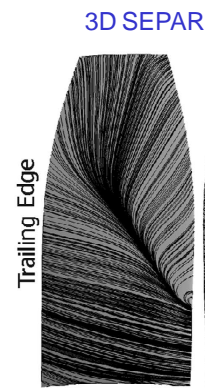

No Re-Pitch

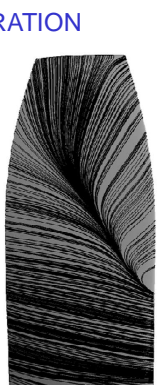

Re-Pitch $-4^{\circ}$

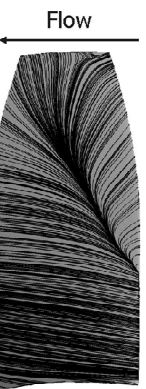

Re-Pitch $-8^{\circ}$

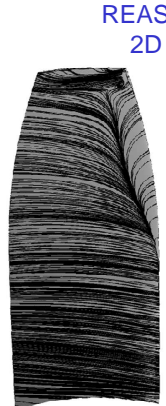

Re-Pitch $-16^{\circ}$
EASONABLY 2D FLOW

ferent take-off conditions for the early design

Figure 15 shows the predicted exit flow from the front rotor at the same operating conditions.

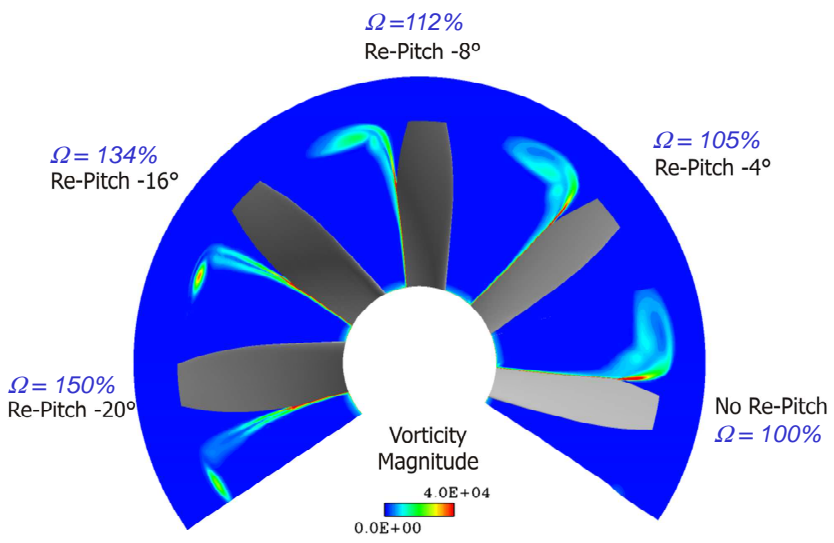

Fig. 15 CFD predicted vorticity contours downstream of the front rotor at different take-off conditions for the early design.

With no re-pitch, $100 \%$ speed, we can see the large loss core, combined with the tip vortex (see also Fig. 9). As the rotor is re-pitched and the separated flow is reduced, the loss core 
is eliminated and the tip vortex becomes more compact. At -20 degrees of re-pitch, the rotational speed is $50 \%$ higher, and the exit flow-field is similar to that seen at cruise: a small, welldefined tip vortex with a distinct wake.

The BEM used above assumed a linear relationship between lift coefficient and incidence and a simple relationship for drag. Figure 16 indicates that the real 3D situation is more complex, especially in this case where there is a significant separated flow.

Figure 16(a) demonstrates how re-pitching reduces the incidence throughout the blade span. The rate of reduction of incidence with re-pitch is almost linear, as suggested by Fig. 13 , but is greater towards the blade tip. The reduction in incidence corresponds to a reduction in lift coefficient and below $r / R=0.7$, the variations in $C_{L}$ and $i$ with radius are very similar and close to linear, as assumed in the BEM. However, a high lift coefficient is not achieved towards the tip of the blade and in the high incidence cases, the lift coefficient in this region collapses to a value of around 0.5. Figure 16(c) shows that modest levels of $C_{L}$ correspond to low $C_{D}$. However, areas where $C_{L}>0.5$ correspond to regions of high drag.

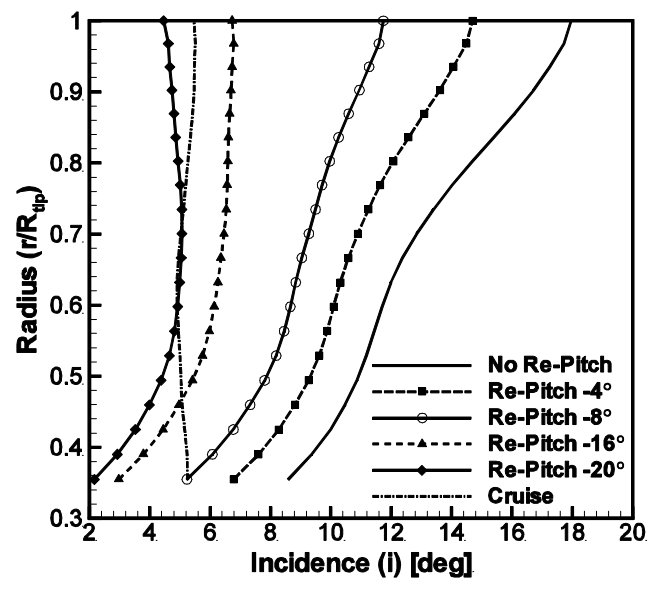

(a) Incidence

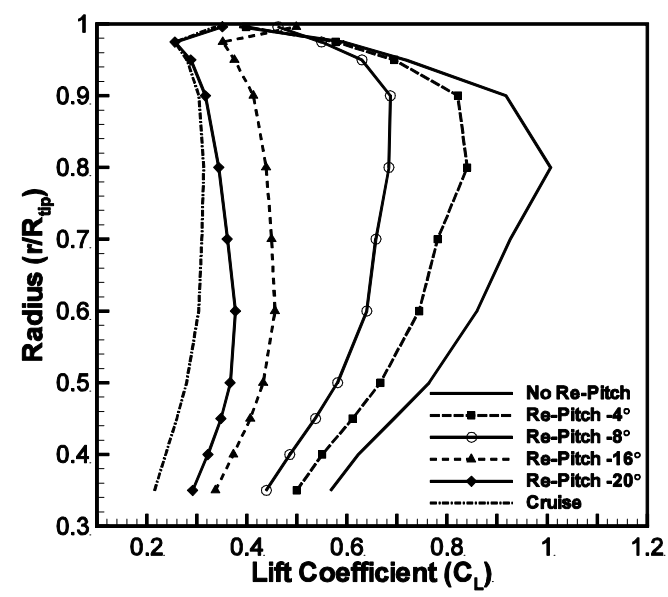

(b) Lift Coefficient

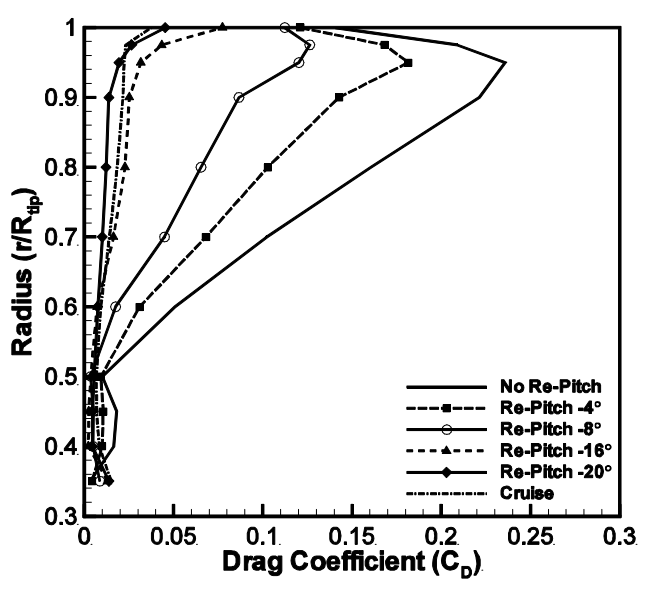

(c) Drag Coefficient

Fig. 16 Radial variations for different take-off re-pitch conditions derived from 3D CFD solutions.

In summary, as the propeller considered is re-pitched and sped up at take-off, the incidence reduces at all radii and the lift coefficient follows this reduction. Once the lift coefficient is low enough, the rotor becomes low drag and low loss, without any significant 3D separations.

\section{Determining the best take-off operating condition}

An open rotor should be operated at take-off to produce a flow-field that is essentially two-dimensional with no significant separations and minimal wakes. However, if a propeller is re-pitched and sped up too much, the high rotational speed can lead to increased rotor alone noise, as found in [3] and [22]. In addition, if a propeller is operated such that $C_{L}$ is much lower than the value at which separation starts to occur, it implies that the same thrust could have been achieved with a higher $C_{L}$ and a smaller propeller (see eqn. (5)).

For a given design, the optimum take-off operating point is therefore when the radial distribution of take-off lift coefficient is as high as possible without causing significant flow separation. At this condition, the flow over the propeller is essentially $2 \mathrm{D}$ with no major $3 \mathrm{D}$ flow features. For the early design considered above, this is somewhere between the re-pitch $-16^{0}$ and $-8^{0}$ cases. For example, in the re-pitch $-16^{0}$ case, $i<7^{0}, C_{L}<$ 0.5 , and $C_{D}<0.05$ at all radii except at the very tip.

Given the required take-off flow-field is essentially 2D, cases with significant 3D flow are not of interest and therefore simpler 2D methods can be used to determine the optimum operating condition. For example, a BEM method can be used to determine the take-off operating condition that gives the required radial distribution of lift coefficient and incidence. This can be combined with aerofoil computations, such as MISES, to determine section loss as a function of incidence to ensure a sufficient margin to flow separation. An example is shown in Fig. 17, which presents a series of MISES predicted loss-loops for the mean-line section of the early propeller design considered above. Each curve shows the predicted variation of loss with incidence for various re-pitch conditions. As the blade is re-pitched and sped up, the loss-loops become narrower and the minimum loss levels increase because the relative Mach num- 
ber increases with blade speed. Also shown are constant takeoff thrust operating points. As expected, as re-pitch is applied, the required incidence reduces and the section loss tends to reduce. In this case, the re-pitch $-12^{0}$ case is found to be low loss for the smallest speed increase, whereas the less re-pitched cases are close to separation. This is consistent with the $3 \mathrm{D}$ CFD results above and demonstrates that a 2D CFD method can be effective in finding a safe take-off operating condition that gives good aerodynamic performance (minimum rotational speed, reduced wakes and no 3D separations).

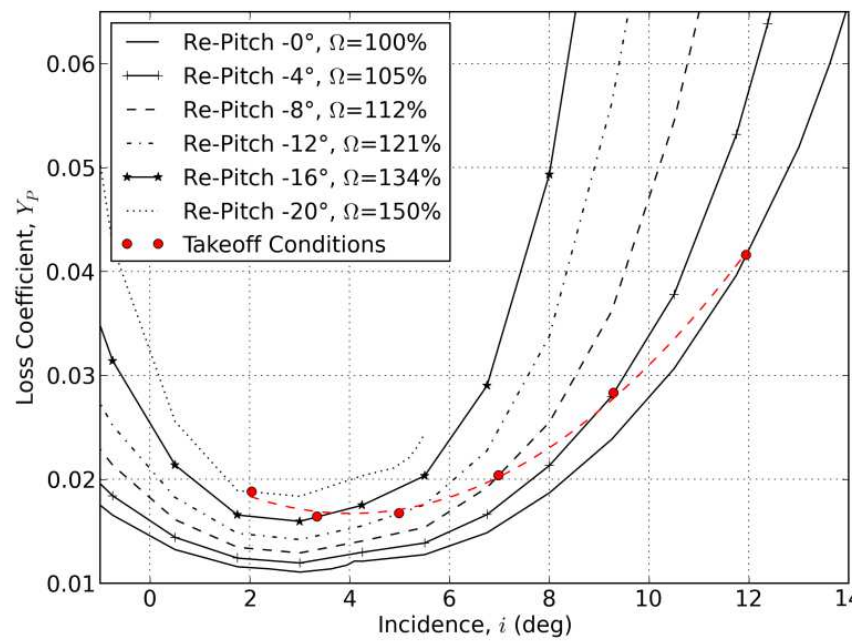

Fig. 17 Predicted variation in section performance for the early design at different re-pitch and speed-up take-off conditions.

\section{CONCLUSIONS}

A power breakdown for an open rotor can be extracted from a 3D steady CFD solution to show the key sources of lost efficiency. For a recent design the rotor losses at both cruise and take-off were found to be significantly lower than for a design from the 1980s. The front rotor loss at take-off is particularly low in the recent design, which indicates small wakes and a flow-field free from large separations. This is expected to lead to reduced rotor-rotor interaction noise.

The rear rotor crop required to reduce interaction noise between the front rotor tip vortex and the rear rotor blade depends on the rotor-rotor axial spacing and angle-of-attack effects. Unsteady 3D CFD is required to resolve this interaction. As crop increases, the losses due to residual swirl increase and a cruise efficiency penalty of around $1 \%$ is typical.

Increased rotor axial spacing can be used to dissipate the front rotor wake before it interacts with the rear rotor, reducing the expected interaction noise. However, minimal benefit is expected for axial spacings beyond two rotor chord lengths.

Rotor sweep has well-known benefits at high speed, but it can also give benefits at take-off by modifying the structure of the front rotor's tip loss core.

Improved blade section aerodynamics have the potential to increase cruise efficiency whilst giving greater incidence tolerance at take-off, thereby enabling operation at higher nondimensional lift without high loss.

At take-off, high incidence onto the front rotor gives higher lift coefficient and if this gets too high the flow separates. The front rotor should be operated with just enough re-pitch and speed-up to eliminate any flow separation. This gives high thrust per unit area for the lowest rotor rotational speeds. The optimum take-off operating condition can be explored in detail with 2D design methods in advance of full 3D CFD.

\section{ACKNOWLEDGEMENTS}

The authors would like to acknowledge the work of the entire open rotor research group in the Whittle Laboratory, Cambridge. In particular, Paul Thomas, Paul Bennett, Tim Newman and Mark Borsuk have all worked with the authors during the time the studies in this paper were completed. The authors are also very grateful for the support from Rolls-Royce plc. Anthony Parry is the lead Rolls-Royce plc contact and his continuous technical advice is greatly appreciated.

\section{REFERENCES}

1. Hanson, D. B. (1980). "Influence of Propeller Design Parameters on Far Field Harmonic Noise in Forward Flight". AIAA J., 18 No. 11:1313-1319

2. Woodward, R. P. and Gordon, E. P. (1988). "Noise of a Model Counter Rotation Propeller With Reduced Aft Rotor Diameter at Simulated Take-off/Approach Conditions (F7/A3)". Technical report, NASA TM-100254.

3. Janardan, B. A. and Gliebe, P. R. (1990). "Acoustic Characteristics of Counterrotating Unducted Fans from Model Scale Tests". J. Aircraft, 27, No. 3:268-275.

4. "The aerodynamics of propellers", Q. R. Wald, Progress in Aerospace Sciences 42 (2006) 85-128

5. Mileshin, V. I., Nyukhtikov, M. A., Orekhov, I. K., Pankov, S. V., and Shchipin, S. K. (2008). "Open Counter-Rotation Fan Blades Optimization Based on 3D Inverse Problem NavierStokes Solution Method With the Aim of Tonal Noise Reduction". ASME Turbo Expo 2008, GT2008-51173

6. Schnell, R., Yin, Y., Voss1, C., and Nicke1, E. (2010), “Assessment and Optimization of the Aerodynamic and Acoustic Characteristics of a Counter Rotating Open Rotor," ASME Paper GT2010-22076.

7. A. Peters and Z. Spakovszky, (2010), "Rotor interaction noise in counter-rotating propfan propulsion systems", GT201022554, ASME Turbo Expo 2010, June 2010, Glasgow, UK

8. A. Zachariadis (2010), "Open Rotor Design for Low Noise", $\mathrm{PhD}$ thesis, University of Cambridge.

9. A. Zachariadis, C. A. Hall, A. B. Parry (2013). "Open Rotor Operation for Improved Aerodynamics and Noise at Take-off", ASME Journal of Turbomachinery, Vol. 135, No. 1.

10. T. Brandvik, C. A. Hall, A. B. Parry (2012). "Angle-of-attack effects on counter-rotating propellers at take-off", GT201269901, ASME Turbo Expo 2012, Copenhagen, Denmark

11. A. Zachariadis and C. A. Hall, (2011). "Application of a Navier-Stokes Solver to the Study of Open Rotor Aerodynamics", ASME Journal of Turbomachinery, Vol. 133, No. 3.

12. Spalart, P. R., and Allmaras, S. R., (1994) "A One-Equation Turbulence Model for Aerodynamic Flows," Recherche Aerospatiale, 1, pp. 5-21.

13. Dittmar, J. H., Gordon, E. B., and Jeracki, R. J. (1988). "The Effect of Front-to-Rear Propeller Spacing on the Interaction Noise at Cruise Conditions of a Model Counterrotation Propeller Having a Reduced Diameter Aft Propeller". Technical report, NASA TM-101329.

14. Majjigi, R. K., Uenishi, K., and Gliebe, P. R. (1989). “An Investigation of Counterrotating Tip Vortex Interaction". Technical report, NASA CR-185135 
15. Schlichting, H. (1960). "Boundary Layer Theory". McGrawHill Book Company, Inc.

16. Parry A B (1988). "Theoretical Prediction of Counter-Rotating Propeller Noise" PhD thesis, Univ. Leeds, UK.

17. Parry, A. B. and Crighton, D. G. (1989). "Prediction of counter rotation propeller noise", AIAA 89-1141, 12th Aeroacoustics Conference, San Antonio, TX.

18. Magliozzi, B., Brown, P., and Parzych, D. (1987). "Acoustic Test and Analysis of a Counterrotating Prop-Fan Model". Technical report, NASA CR-179590.

19. Metzger, F. B., and Rohrbach, C., 1986, "Benefits of Blade Sweep for Advanced Turboprops," J. Propul., 2(6), pp. 534540.

20. Rohrbach, C., Metzger, F. B., Black, D. M., and Ladden, R. M. (1982). "Evaluation of Wind Tunnel Performance Testings of an Advanced 45 degree Swept Eight-Bladed Propeller at Mach Numbers from 0.45 to 0.85 ". Technical report, NASA CR-3505.

21. Parry, A. B. (1995) "The effect of blade sweep on the reduction and enhancement of propeller noise", J. Fluid Mech., vol. 293, pp. 181-206.

22. Dittmar, J. H. and Stang, D. B. (1987). "Cruise Noise of the 2/9th Scale Model of the Large-Scale Advanced Propfan (LAP) Propeller, SR-7A". Technical report, NASA TM-100175

23. Woodward, R. P. (1987). "Noise of a Model High Speed Counterrotation Propeller at Simulated Take-off/Approach Conditions (F7/A7)". Technical report, NASA TM-100206.

24. Goodhand, M. N., and Miller, R. J., (2010), "Compressor Leading Edge Spikes: A New Performance Criterion,” ASME J. Turbomach., 133 , p. 021006

25. Drela, M. and Youngren, H. (1998). "A User's Guide to MISES 2.53". MIT Computational Aerospace Sciences Laboratory.

26. Glauert, H. (1926), "The Elements of Aerofoil and Airscrew Theory", Cambridge University Press.

\section{APPENDIX - MECHANICAL POWER LOSSES}

The open rotor efficiency can be expressed in terms of lost mechanical power. Consider the flow through the open rotor from far upstream (station 0) to far downstream in the open rotor jet (station $\mathrm{j}$ ). The following simplifying assumptions are made: (i) the flow downstream of the open rotor is uniform (ii) atmospheric pressure applies across the downstream jet (iii) there is no core flow. In this case the enthalpy-entropy diagram for the open rotor is as shown in Fig. A.1.

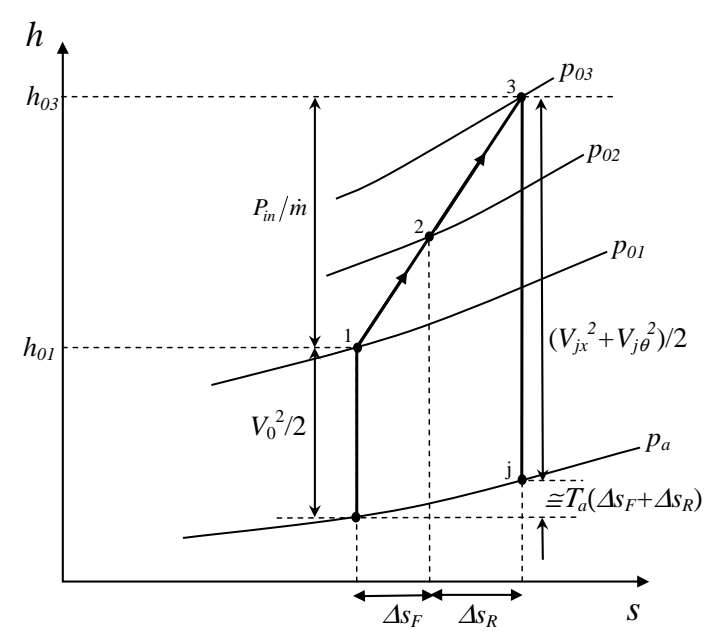

Fig. A.1: Enthalpy-entropy diagram for an open rotor

Applying conservation of energy,

$$
\frac{P_{i n}}{\dot{m}}+\frac{1}{2} V_{0}^{2}=\frac{1}{2}\left(V_{j x}^{2}+V_{j \theta}^{2}\right)+T_{a}\left(\Delta s_{F}+\Delta s_{R}\right)
$$

This can be re-written as:

$$
\frac{P_{i n}}{\dot{m}}=V_{0}\left(V_{j x}-V_{0}\right)+\frac{1}{2}\left(V_{j x}-V_{0}\right)^{2}+\frac{1}{2} V_{j \theta}^{2}+T_{a}\left(\Delta s_{F}+\Delta s_{R}\right)
$$

Hence,

$$
\frac{\dot{m}\left(V_{j x}-V_{0}\right) V_{0}}{P_{i n}}=1-\frac{\dot{m}}{P_{i n}}\left\{\frac{1}{2}\left(V_{j x}-V_{0}\right)^{2}+\frac{1}{2} V_{j \theta}^{2}+T_{a}\left(\Delta s_{F}+\Delta s_{R}\right)\right.
$$

From conservation of momentum,

$$
\begin{gathered}
\eta=\frac{X V_{0}}{P_{i n}}=\frac{\dot{m}\left(V_{j x}-V_{0}\right) V_{0}}{P_{i n}} \\
\Rightarrow \eta=1-\frac{\dot{m}}{P_{i n}}\left\{\frac{1}{2}\left(V_{j x}-V_{0}\right)^{2}+\frac{1}{2} V_{j \theta}^{2}+T_{a}\left(\Delta s_{F}+\Delta s_{R}\right)\right\} \\
\hline
\end{gathered}
$$

Note also that:

$$
\frac{1}{2} \frac{\dot{m}}{P_{i n}}\left(V_{j x}-V_{0}\right)^{2}=\frac{X^{2}}{2 \dot{m} P_{i n}}=\frac{P_{i n} \eta^{2}}{2 \dot{m} V_{0}^{2}}
$$

